University of Wollongong

Research Online

Faculty of Engineering and Information

Faculty of Engineering and Information

Sciences - Papers: Part B

Sciences

2019

\title{
A fringe projection profilometry scheme based on embedded speckle patterns and robust principal component analysis
}

Yiwei Zhang

University of Wollongong, yz831@uowmail.edu.au

Jun Tong

University of Wollongong, jtong@uow.edu.au

Jiangtao Xi

University of Wollongong, jiangtao@uow.edu.au

Chengpu Duan

University of Wollongong, cd093@uowmail.edu.au

Yanguang Yu

University of Wollongong, yanguang@uow.edu.au

See next page for additional authors

Follow this and additional works at: https://ro.uow.edu.au/eispapers1

Part of the Engineering Commons, and the Science and Technology Studies Commons

Research Online is the open access institutional repository for the University of Wollongong. For further information contact the UOW Library: research-pubs@uow.edu.au 


\title{
A fringe projection profilometry scheme based on embedded speckle patterns and robust principal component analysis
}

\author{
Abstract \\ 2019 SPIE. Phase unwrapping is one of the key steps for fringe projection profilometry (FPP)-based 3D \\ shape measurements. Conventional spatial phase unwrapping schemes are sensitive to noise and \\ discontinuities, which may suffer from low accuracies. Temporal phase unwrapping is able to improve the \\ reliability but often requires the acquisition of additional patterns, increasing the measurement time or \\ hardware costs. This paper introduces a novel phase unwrapping scheme that utilizes composite \\ patterns consisting of the superposition of standard sinusoidal patterns and randomly generated \\ speckles. The low-rankness of the deformed sinusoidal patterns is studied. This is exploited together with \\ the sparse nature of the speckle patterns and a robust principal component analysis (RPCA) framework is \\ then deployed to separate the deformed fringe and speckle patterns. The cleaned fringe patterns are used \\ for generating the wrapped phase maps using the standard procedures of phase shift profilometry (PSP) \\ or Fourier Transform profilometry (FTP). Phase unwrapping is then achieved by matching the deformed \\ speckle patterns that encode the phase order information. In order to correct the impulsive fringe order \\ errors, a recently proposed postprocessing step is integrated into the proposed scheme to refine the \\ phase unwrapping results. The analysis and simulation results demonstrate that the proposed scheme \\ can improve the accuracy of FPP-based 3D shape measurements by effectively separating the fringe and \\ speckle patterns. \\ Disciplines \\ Engineering | Science and Technology Studies

\section{Publication Details} \\ Y. Zhang, J. Tong, J. Xi, C. Duan, Y. Yu, Q. Guo \& L. Lu, "A fringe projection profilometry scheme based on \\ embedded speckle patterns and robust principal component analysis," Proceedings of SPIE - The \\ International Society for Optical Engineering, vol. 11205, pp. 1-7, 2019.
}

\section{Authors}

Yiwei Zhang, Jun Tong, Jiangtao Xi, Chengpu Duan, Yanguang Yu, Qinghua Guo, and Lei Lu 


\title{
A Fringe Projection Profilometry Scheme Based on Embedded Speckle Patterns and Robust Principal Component Analysis
}

\author{
Yiwei Zhang ${ }^{\mathrm{a}}$, Jun Tong ${ }^{\mathrm{a}}$, Jiangtao Xi ${ }^{*}$, Chengpu Duan ${ }^{\mathrm{a}}$, Yanguang Yu ${ }^{\mathrm{a}}$, Qinghua Guo and Lei Lu ${ }^{\mathrm{b}}$ \\ ${ }^{a}$ School of Electrical, Computer and Telecommunications Engineering, University of Wollongong, \\ Northfields Ave, Wollongong, NSW 2522, Australia \\ ${ }^{b}$ College of Information Science and Engineering, Henan University of Technology, Zhengzhou, \\ 450001, China \\ jiangtao@uow.edu.au \\ yz831@uowmail.edu.au
}

\begin{abstract}
Phase unwrapping is one of the key steps for fringe projection profilometry (FPP)-based 3D shape measurements. Conventional spatial phase unwrapping schemes are sensitive to noise and discontinuities, which may suffer from low accuracies. Temporal phase unwrapping is able to improve the reliability but often requires the acquisition of additional patterns, increasing the measurement time or hardware costs. This paper introduces a novel phase unwrapping scheme that utilizes composite patterns consisting of the superposition of standard sinusoidal patterns and randomly generated speckles. The low-rankness of the deformed sinusoidal patterns is studied. This is exploited together with the sparse nature of the speckle patterns and a robust principal component analysis (RPCA) framework is then deployed to separate the deformed fringe and speckle patterns. The cleaned fringe patterns are used for generating the wrapped phase maps using the standard procedures of phase shift profilometry (PSP) or Fourier Transform profilometry (FTP). Phase unwrapping is then achieved by matching the deformed speckle patterns that encode the phase order information. In order to correct the impulsive fringe order errors, a recently proposed postprocessing step is integrated into the proposed scheme to refine the phase unwrapping results. The analysis and simulation results demonstrate that the proposed scheme can improve the accuracy of FPP-based 3D shape measurements by effectively separating the fringe and speckle patterns.
\end{abstract}

Keywords: Fringe Projection Profilometry; Robust Principal Component Analysis; Phase Unwrapping

\section{INTRODUCTION}

Fringe projection profilometry (FPP) has been widely applied to three-dimensional (3D) shape measurements due to its non-contact nature and superior performance in measurement speed and accuracy [1-4]. A typical FPP system consists of a projector and a camera. During the measurement, one or several periodic fringe patterns are projected onto the object; then, the patterns deformed by the uneven surface of the objects are captured by a camera from a different angle. By analyzing the difference between the reference images and deformed images the 3D shape information of the objects can be retrieved. However, traditional fringe analysis methods can only provide wrapped phase maps, which have $2 \pi$ discontinuities and can not be directly used for 3D construction. Therefore, phase unwrapping procedures are required to unwrap the phase in order to eliminate the discontinuities and to obtain the true phase maps [1-5].

Over the past decades, many phase unwrapping algorithms have been developed, which may be classified into two categories: spatial phase unwrapping and temporal phase unwrapping [3-4]. Spatial phase unwrapping is based on the analysis of neighboring pixels on a single wrapped phase map. It often fails to determine the fringe order and recover the absolute phase for spatially isolated objects [3]. In contrast, temporal phase unwrapping is able to recover the absolute phase for objects with abrupt surface changes by utilizing additional patterns. Typical examples include gray-code pattern projection [6] and multi-frequency approaches [7, 8]. However, the requirement of extra patterns may slow down the measurement speed.

In order to achieve fast and reliable phase unwrapping, approaches based on composite patterns which embed auxiliary signals into the fringe patterns have been proposed. In these approaches, the fringe order information is encoded using structural markers or specially designed patterns. Budianto et al [9] embed several strip markers within a single fringe pattern to facilitate phase unwrapping and retrieve the marker cue through the dual-tree complex wavelet transform. 
Similarly, Hu et al [10] propose a two-marker approach by embedding two specially designed markers with opposite intensity, which can enhance the absolute phase retrieval. However, these methods can only be used for reconstructing simple objects and may perform poorly for complex scenes. Zhang et al [11] propose to fuse speckle-like signal into the three-step phase shifting method. The sinusoidal fringes are used for fringe analysis, while the randomly distributed speckle signal is used to determine the fringe order. However, their method does not separate the speckle patterns and fringe patterns when determining the fringe order. This can lead to errors on the estimation of fringe order and further affect the final reconstruction results.

In this work, we propose a novel phase unwrapping scheme that utilizes speckle-embedded fringe patterns. To achieve this, we exploit the low-rank property of the fringe signal and sparse nature of the speckle signal and apply robust principal component analysis (RPCA) to effectively separate the fringe patterns and speckles. We then retrieve the wrapped phase from the recovered fringe patterns using Fourier transform profilometry (FTP), and determine the fringe orders based on the recovered speckle pattern. To further eliminate the fringe order errors, a recently proposed fringe order correction algorithm is integrated into the scheme. Simulation results verify the effectiveness of the proposed method.

\section{THE PROPOSED METHOD}

\subsection{System Model}

We consider a single-shot measurement setting, which may be useful for achieving fast measurement. We adopt the following composite pattern which is the superposition of a pseudo-random speckle pattern and an ordinary sinusoidal fringe pattern:

$$
I_{p}(x, y)=A+B \cos (2 \pi f x)+C Z(x, y),
$$

where $(x, y)$ give the coordinates of the pixel, $A$ and $B$ represent the background intensity and intensity modulation, respectively, for the sinusoidal fringe pattern with a spatial frequency of $f . Z(x, y) \in\{0,1\}$ and $C$ control the distribution and intensity of the speckle signal, respectively. Following [11], we adopt the following rules while generating the speckle patterns in order to guarantee the distinguishability within a local window: (1) The speckle is simulated as white dots with size $M \times M$; (2) In each equivalent area of $3 \times 3$ dots, only one dot is white; (3) No two white dots are adjacent in their eight neighborhoods. The fringe order for each pixel is uniquely encoded by a sufficiently large patch of the speckle pattern centered at that pixel. When the designed pattern is projected onto the measured objects, the captured image is expressed as:

$$
I_{c}(x, y)=\alpha(x, y)\left\{A+B \cos [\phi(x, y)]+C Z^{\prime}(x, y)\right\}+\beta(x, y),
$$

where $Z^{\prime}(x, y)$ denotes the deformed speckle pattern, $\alpha(x, y)$ is the reflectivity of the object, $\beta(x, y)$ is the ambient light and $\phi(x, y)$ is the phase to be solved. For single-shot measurements, we often apply FTP by converting the deformed pattern into the frequency domain. Then the fundamental frequency is extracted by appropriate band-pass filtering. From (2), the speckle and fringe patterns are mixed and thus the standard FTP method cannot be directly applied. In the following, we propose a scheme to separate the speckle and fringe patterns using RPCA. We can then recover the wrapped phase using FTP and perform phase unwrapping by speckle correlation. In addition, we also introduce a recently proposed method to correct the fringe order errors.

\subsection{Pattern Analysis Based on Robust Principal Component Analysis (RPCA)}

The deformed pattern in (2), which is the superposition of the deformed speckle pattern and deformed fringe pattern, can be rewritten as

$$
\begin{gathered}
I_{c}(x, y)=S(x, y)+L(x, y)+\beta(x, y), \\
S(x, y)=\alpha(x, y) C Z^{\prime}(x, y), \\
L(x, y)=\alpha(x, y)(A+B \cos [\phi(x, y)]),
\end{gathered}
$$

In the above, $S(x, y)$ denotes the deformed speckle pattern and $L(x, y)$ is the deformed fringe pattern. We propose to separate these patterns such that the performance of reconstruction can be improved. According to the process of generating the speckle patterns as described above, the speckle pattern $S(x, y)$ is sparse, with about $11.1 \%$ of the pixels assuming nonzero grey values. For many applications, the fringe pattern $L(x, y)$ is also low-rank. Figure 1 demonstrates this property where the singular values of an example deformed fringe pattern with size $1000 \times 1000$ are shown. It can be observed that the singular values decrease rapidly and a small number, e.g., 10, of singular values can capture most 
of the information of the fringe pattern. This number is significantly smaller than the size of $L(x, y)$ and thus $L(x, y)$ can be regarded as a low-rank matrix.

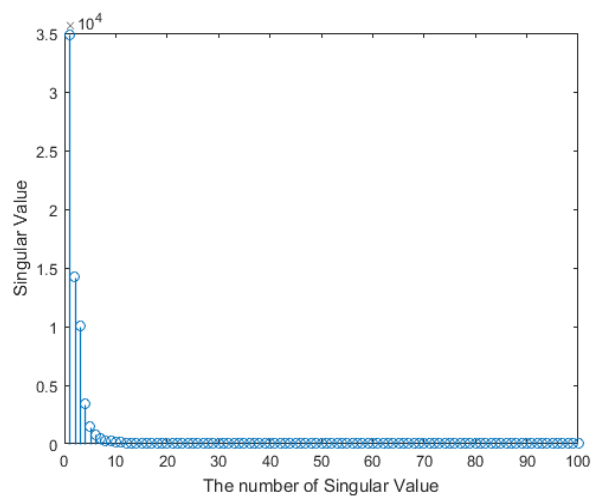

Figure 1. Singular value distribution of a simulated deformed fringe pattern with size $1000 \times 1000$

Exploiting the above properties of $L$ and $S$, it is possible to separate the fringe pattern and speckle pattern from the deformed image $I_{c}$ by using RPCA. However, the original PRCA problem is NP-hard and thus not trackable [12-13]. Following [12-14], we relax the problem by using the $l_{1}$ norm and nuclear norm to induce low-rankness and sparsity. The problem is now formulated as:

$$
\min _{S, L} \gamma\|S\|_{l_{1}}+\|L\|_{*}, \quad \text { s.t. } \quad S+L=I_{c}
$$

where $\|\cdot\|_{l_{1}}$ is the $l_{1}$ norm, defined as the sum of the absolute value of the elements in $S,\|\cdot\|_{*}$ is the nuclear norm, defined as the sum of all the singular value of $L$, and $\gamma>0$ is a constant to balance low-rankness and sparsity. Different algorithms can be applied to approximately solve (6). We adopt the alternating direction method (ADM), which minimizes the following augmented Lagrangian function:

$$
L_{\mathcal{A}}(S, L, E)=\gamma\|S\|_{l_{1}}+\|L\|_{*}-\left\langle E, S+L-I_{c}\right\rangle+\frac{\lambda}{2}\left\|S+L-I_{c}\right\|^{2},
$$

where $E \in \mathcal{R}^{m \times n}$ is the multiplier of the linear constraint of (6); $\lambda>0$ is the penalty parameter for the violation of linear constraint and $\langle\cdot\rangle$ represents the standard trace inner product. The solution can be found iteratively. Let $P_{\Omega_{\infty}^{\gamma / \lambda}}$ denote the Euclidean projection onto $\Omega_{\infty}^{\gamma / \lambda}=\left\{X \in \mathcal{R}^{n \times n} \mid-\gamma / \lambda \leq X_{i j} \leq \gamma / \lambda\right\}, k$ the number of iteration, and $\left(S^{k}, L^{k}, E^{k}\right)$ the current solution. The ADM updates the solution using the following steps [14]:

1. Generate $S^{k+1}=\frac{1}{\lambda} E^{k}-L^{k}+I_{c}-P_{\Omega_{\infty}^{\gamma / \lambda}}\left(\frac{1}{\lambda} E^{k}-L^{k}+I_{c}\right)$

2. Generate $L^{k+1}=U^{k+1} \operatorname{diag}\left(\max \left\{\sigma_{i}^{k+1}-\frac{1}{\lambda}, 0\right\}\right)\left(V^{k+1}\right)^{T}$, where $U^{k+1}, V^{k+1}$ and $\left\{\sigma_{i}^{k+1}\right\}$ are generated by the singular value decomposition (SVD) of $I_{c}-S^{k+1}+\frac{1}{\lambda} E^{k}$

3. Update the multiplier $E^{k+1}=E^{k}-\lambda\left(S^{k+1}+L^{k+1}-I_{c}\right)$

We can terminate ADM by setting an appropriate stopping criterion: The iteration stops when $\frac{\left\|\left(S^{k+1}, L^{k+1}\right)-\left(S^{k}, L^{k}\right)\right\|_{F}}{\left\|\left(S^{k}, L^{k}\right)\right\|_{F} F^{1}} \leq$ $10^{-6}$.

Once the deformed fringe and speckle patterns are separated, we can retrieve the phase map from image $L$ by using FTP. However, the FTP-based approach can only provide wrapped phase value ranging from $-\pi$ to $\pi$, which has $2 \pi$ discontinuities. Thus, phase unwrapping is needed to eliminate the phase ambiguity as:

$$
\Phi(x, y)=\phi(x, y)+2 \pi \times d(x, y)
$$

where $\Phi(x, y)$ denotes the absolute phase and $d(x, y)$ the fringe order. Spatial phase unwrapping methods may be used. However, they may suffer from error propagation and fail to determine the fringe orders correctly for spatially isolated objects. 


\subsection{Enhanced Speckle Correlation-Based Phase Unwrapping}

In this subsection, we introduce a speckle correlation-based image matching approach to unwrap the phase. We assume that the deformed speckle patch and the corresponding reference speckle patch share nearly the same local speckle distribution. The fringe order can then be found by maximizing the following normalized correlation coefficient:

$$
\operatorname{corr}=\frac{\sum_{x, y}\left(S_{r}(x, y)-\overline{S_{r}}\right)\left(S_{d}(x, y)-\overline{S_{d}}\right)}{\sqrt{\sum_{x, y}\left(S_{r}(x, y)-\overline{S_{r}}\right)^{2}} \sqrt{\sum_{x, y}\left(S_{d}(x, y)-\overline{S_{d}}\right)^{2}}}
$$

where $S_{r}$ and $S_{d}$ are the same-size patches captured from the reference speckle image and the deformed speckle image (separated by using RPCA), respectively, $\overline{S_{r}}$ and $\overline{S_{d}}$ are the average intensity of $S_{r}$ and $S_{d}$. The fringe order is an integer number within $[0, K-1]$, where $K$ denotes the number of candidate fringe orders. Thus, for each pixel in the deformed image, (9) is computed for $K$ times, each time with a patch in the reference pattern whose center pixel has the same wrapped phase.

The above method has similarity to [11] in using image correlation to identify the fringe order. However, we have utilized the cleaned speckle pattern with the influence from the fringe patterns eliminated, while [11] uses directly the composite pattern where the speckle and fringe patterns are mixed. We have observed from our simulations that the proposed approach can significantly improve the accuracy of fringe order recovery as compared with the approach without separating the two components.

Let us consider the fringe order sequence $d(x, y)$ retrieved using speckle correlation. Ideally, $d(x, y)$ should exhibit a stepwise increase with respect to the direction vertical to the fringes (i.e., $x$-axis), and $d(x, y)$ increases by 1 on the boundary of any two adjacent fringes. However, speckle correlation-based phase unwrapping can still suffer from errors in the fringe order due to the deformation of the speckle pattern and noise, which result in unwrapped phase errors and errors in the ultimate 3D shape reconstruction. In speckle correlation-based phase unwrapping, the fringe order of each pixel is recovered independently. Therefore, the fringe order error of one specific pixel will not propagate into the adjacent pixels. In light of this, we apply a recently proposed fringe order correction method to remove errors [15].

1. Row by row, divide the recovered fringe order map into several steps based on phase jumps in the wrapped phase.

2. Counting the number of pixels with different fringe order values in each step, select the fringe order value with the highest frequency. This fringe order value is selected as the true fringe order value of this step.

\section{SIMULATION RESULTS}

In order to verify the feasibility of the proposed method, numerical simulations are carried out. We use a hemisphere and double hemisphere as the measured objects, respectively. Gaussian noise with a variance of $\rho$ and a mean of zero is added to the generated pattern and we assume perfect calibration. The signal to noise ratio SNR $\triangleq \frac{1}{\rho M N} \sum_{x=1}^{M} \sum_{y=1}^{N}\left|s_{x, y}\right|^{2}$ is set as $25 \mathrm{~dB}$ with the image size $M \times N=1000 \times 1000$. (We assume that the effect of reflectivity $\alpha(x, y)$ can be ignored.)

We first demonstrate in Fig. 2 the feasibility of applying the RPCA method for separating the patterns. It is seen that we are able to separate the fringe pattern and the speckle pattern in the deformed image by taking advantage of the low-rank structure of the fringe signal and the sparse nature of the speckle signal.

In order to balance the computational complexity and performance, speckle image patches of size $27 \times 27$ are used for correlation analysis. Fig. 3(a)-(b) show that our method can effectively reconstruct the object with high accuracy. To demonstrate the performance of reconstructing isolated objects of our method, we also measure a double-hemisphere object and the results are shown in Fig. 3(c)-(d). It is seen that the proposed method can reconstruct the entire scene with single projection. 


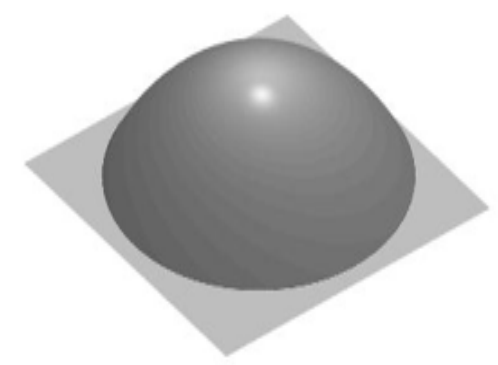

(a)

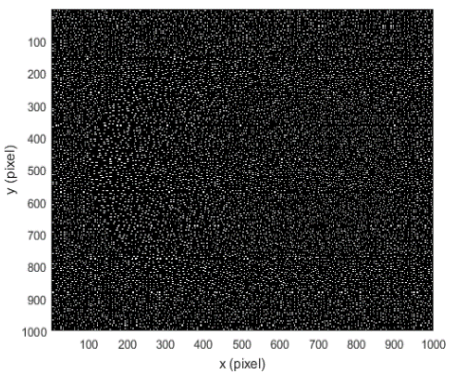

(c)

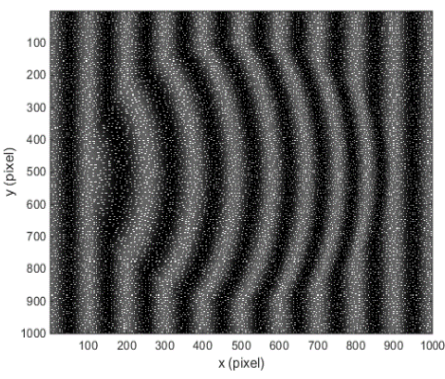

(b)

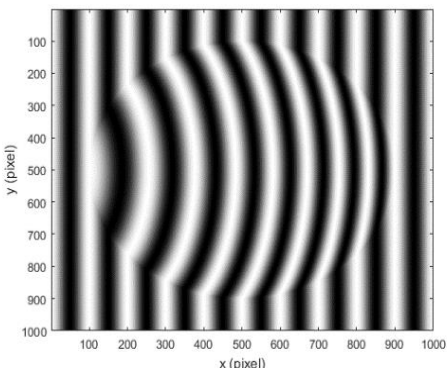

(d)

Figure 2. Separation of the fringe pattern and the speckle pattern by using RPCA. (a) Simulated hemisphere. (b) Speckle-embedded image of hemisphere. (c) Separated speckle pattern. (d) Separated fringe pattern.

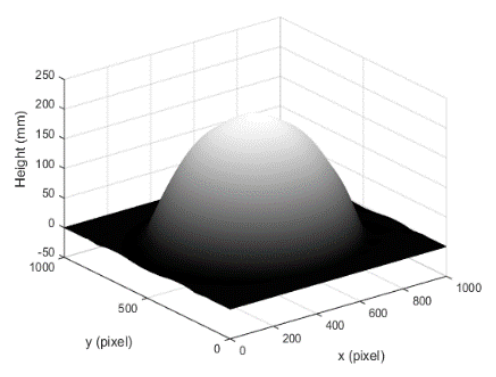

(a)

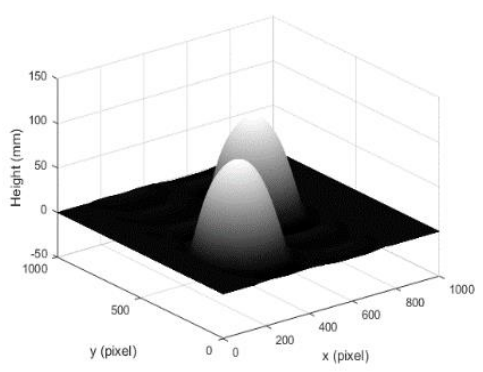

(c)

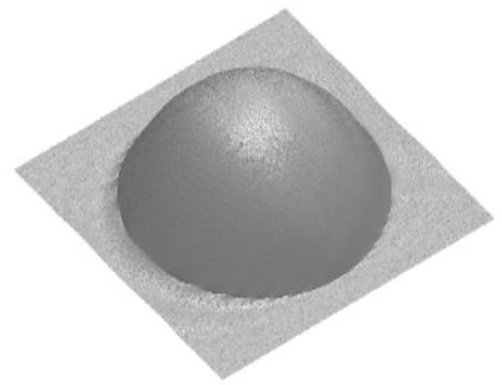

(b)

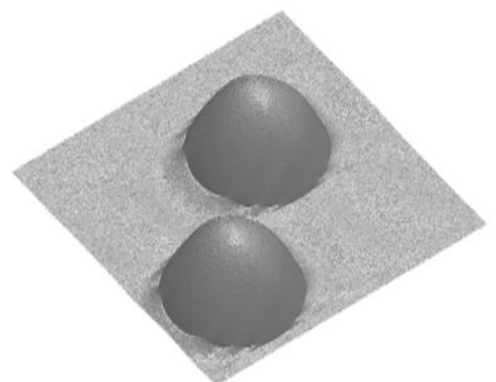

(d)

Fig. 3. Simulation results for measuring the 3D shape of a hemisphere and a double-hemisphere. (a) Reconstructed sphere with Mesh display (b) Reconstructed hemisphere surface (c) Reconstructed double-hemisphere with Mesh display (d) Reconstructed double hemisphere surface

The effectiveness of the fringe order correction scheme is also demonstrated in Fig. 4. Fig. 4(a) indicates that there are noticeable errors in the fringe orders after applying speckle correlation-based phase unwrapping. Fig. 4(b) depicts the fringe orders of the recovered absolute phase map. Using the correction method, we successfully correct the fringe order 
errors and the corrected fringe orders are shown in Fig. 4(c) and (d). Based on the corrected fringe order maps, we are able to obtain the smoothed absolute phase map for further 3D reconstruction.

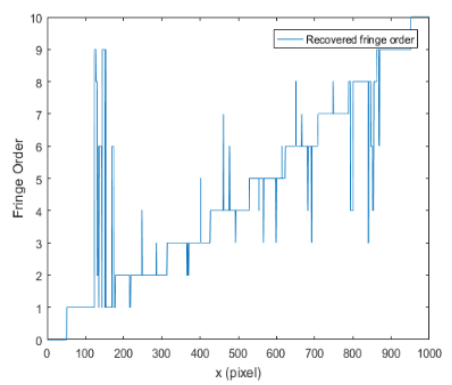

(a)

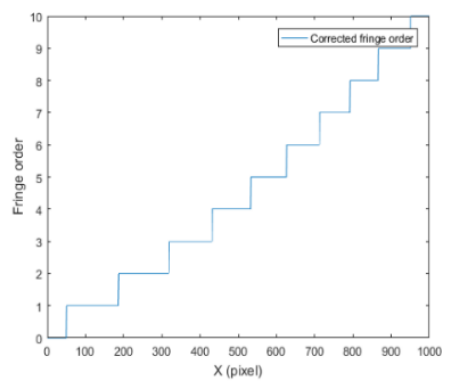

(c)

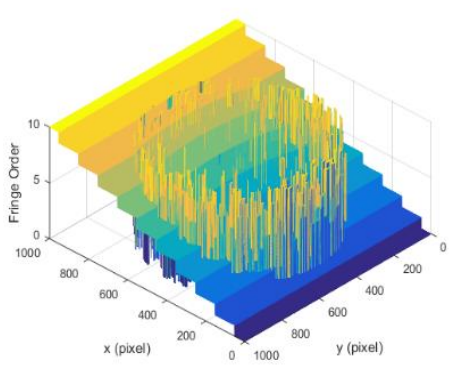

(b)

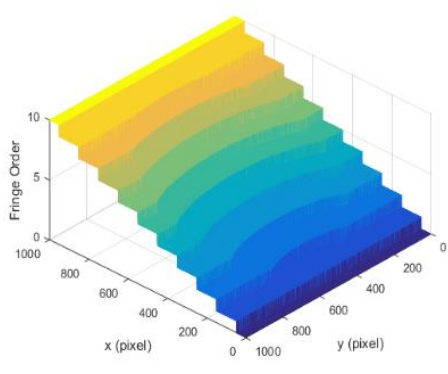

(d)

Figure 4. Fringe order correction results on the section $y=400$. (a) Recovered fringe order using speckle correlation (b) Fringe order of the hemisphere without correction (c) Corrected fringe order after employing the correction (d) Corrected fringe order of the hemisphere.

\section{CONCLUSION}

In this paper, we propose a FPP technique based on speckle-embedded patterns. RPCA is applied to effectively separate the fringe and speckle patterns, which enables wrapped phase retrieval and phase unwrapping to be achieved using only a single projection of the composite pattern. A recently proposed fringe order correction method is adopted to efficiently correct the fringe order errors. The effectiveness of the proposed scheme has been verified by numerical simulations.

\section{REFERENCES}

[1] S. Zhang, "Recent progresses on real-time 3D shape measurement using digital fringe projection techniques," Optics and Lasers in Engineering. 48, 149-158 (2010).

[2] C. Zuo, S. Feng, L. Huang, T. Tao, W. Yin, and Q. Chen, "Phase shifting algorithms for fringe projection profilometry: A review," Optics and Laser in Engineering. 109, 23-59 (2018).

[3] C. Zuo, L. Huang, M. Zhang, Q. Chen, and A. Asundi, "Temporal phase unwrapping algorithms for fringe projection profilometry: A comparative review," Optics and Laser in Engineering. 84, 84-103 (2016).

[4] S. Zhang, "Absolute phase retrieval methods for digital fringe projection profilometry: A review," Optics and Laser in Engineering. 107, 28-37 (2018).

[5] Zhao M, Huang L, Zhang Q, Su X, Asundi A, Kemao Q, Quality-guilded phase unwrapping technique: comparison of quality maps and guilding strategies. Applied Optics. 50(33), 6214-6224 (2011).

[6] Q. Zhang, X. Su, L. Xiang, and X. Sun, "3-D shape measurement based on complementary Gray-code light," Optics and Lasers in Engineering. 50(4), 574-579 (2012). 
[7] K. Liu, Y. Wang, D. L. Lau, Q. Hao, and L. G. Hassebrook, "Dual frequency pattern scheme for high-speed 3-D shape measurement,” Optics Express. 18(5), 5229-5244 (2010).

[8] Y. Ding, J. Xi, Y. Yu, W. Cheng, S. Wang, and J. F. Chicharo, "Frequency selction in absolute phase maps recovery with two requency projection fringes," Optics Express. 20(12), 13238-13251 (2012).

[9] B. Budianto, P. Lun, and T.-C. Hsung, "Marker encoded fringe projection profilometry for efficient 3d model acquisition," Applied Optics. 53, 7442-7453 (2014).

[10] Y. Hu, Q. Chen, Y. Zhang, S. Feng, T. Tao, H. Li, W. Yin, and C. Zuo, "Dynamic microscopic 3d shape measurement based on marker-embedded fourier transform profilometry," Applied Optics. 57, 772-780 (2018).

[11] Y. Zhang, Z. Xiong, and F. Wu, "Unambiguous 3D measurement from speckle-embedded fringe," Applied Optics. 52(32), 7797-7805 (2013).

[12] V. Chandrasekaran, S. Sanghavi, P. A. Parrilo, and A. S. Willsky, "Rank-sparsity incoherence for matrix decomposition,” SIAM J. Optim. 21(2), 572-596 (2011).

[13] E. J. Candes, X. D. Li, Y. Ma, and J. Wright. "Robust principal component analysis?" J. ACM, 58(3), (2011).

[14] X. Yuan and J. Yang, "Sparse and low-rank matrix decomposition via alternating direction methods," Pacific J. Opt. 9(1), 167-180, (2009).

[15] Y. Ding, K. Peng, L. Lu, K. Zhong, and Z. Zhu, "Simplified fringe order correction for absolute phase maps recovered with multiple-spatial-frequency fringe projections,” Meas. Sci. Technol. 28(2), 025203 (2017). 